This item was submitted to Loughborough's Research Repository by the author.

Items in Figshare are protected by copyright, with all rights reserved, unless otherwise indicated.

\title{
Models for estimation of creep forces in the wheel/rail contact under varying adhesion levels
}

PLEASE CITE THE PUBLISHED VERSION

http://dx.doi.org/10.1080/00423114.2014.901541

PUBLISHER

Taylor \& Francis

VERSION

AM (Accepted Manuscript)

\section{PUBLISHER STATEMENT}

This work is made available according to the conditions of the Creative Commons Attribution-NonCommercialNoDerivatives 4.0 International (CC BY-NC-ND 4.0) licence. Full details of this licence are available at: https://creativecommons.org/licenses/by-nc-nd/4.0/

\section{LICENCE}

CC BY-NC-ND 4.0

\section{REPOSITORY RECORD}

Hubbard, Peter, Christopher Ward, Roger Dixon, and Roger Goodall. 2014. "Models for Estimation of Creep Forces in the Wheel/rail Contact Under Varying Adhesion Levels". Loughborough University. https://hdl.handle.net/2134/24518. 


\title{
RESEARCH ARTICLE
}

\section{Models for Estimation of Creep Forces in the Wheel/Rail Contact Under Varying Adhesion Levels}

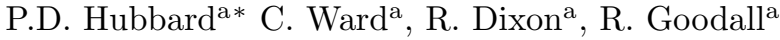

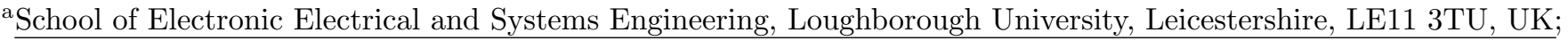

(October 2013)

\begin{abstract}
Areas of extremely low adhesion between the wheel and rail can cause critical problems in traction and braking that can manifest in issues such as signals being passed at danger. There is currently a lack of real time information regarding the state and location of low adhesion areas across rail networks.

The study presented here examines the scientific challenges of understanding the change in vehicle running dynamics with variations in adhesion using the latest thinking of adhesion at micro-slip. This understanding supports the generation of suitable low-order dynamic models for use with a model-based estimator that infers adhesion levels in the wheel/rail contact using signals from modest-cost sensors that could be fitted to in-service vehicles.

This paper presents verification of this technique by using simulated inertial measurement produced from a high fidelity multibody simulation in a series of 'blind' tests.
\end{abstract}

Keywords: Railways; Vehicle Dynamics; Low Adhesion; Kalman Filtering; Creep Force

\section{Introduction}

The level of adhesion between the wheel and the rail is of critical importance for the safe and punctual running of any railway. Areas of extremely low adhesion (typically $\mu<0.07$ ) can cause traction issues (wheel spin/slip) and perhaps more importantly braking issues (wheel slide) that can manifest in increased operational risks such as track signals being passed at danger, station stops being missed, and network wide disruption due to the application of defensive driving techniques [1]. There is currently a lack of real time information about the state of adhesion in the wheel/rail interface. As a result, studies such as [2] investigated methods that utilise inertial sensing mounted on service vehicles to infer this information. The study presented here looks at the scientific challenges of selecting suitable models (simulation and design) for a previously published technique [3] that uses advanced model-based filtering of inertial measurements (accelerometers and gyroscopes) to estimate the creep forces in the wheel/rail contact. Changes in the creep force estimates in normal running conditions can then be used to infer adhesion levels.

The research presented in this article has been conducted as part of a wider project to investigate methods of detecting low adhesion by monitoring lateral and yaw running dynamics. This research is a progression from a feasibility study that proved the concept of inferring adhesion levels using advanced methods to process measurements from low-cost inertial sensors $[4,5]$. It was proven that changes in

*Corresponding author. Email: p.d.hubbard3@lboro.ac.uk 
Table 1. Quantitative Description of Adhesion Levels

\begin{tabular}{cc}
\hline Qualitative Description & Coefficient of Friction $(\mu)$ \\
\hline Dry & 0.560 \\
Wet & 0.320 \\
Low & 0.072 \\
Very Low & 0.038 \\
\hline
\end{tabular}

adhesion level results in a different dynamic vehicle response. This variation in motion is able to be modelled using only the lateral and yaw motions of a vehicle as these capture the major responses to track irregularities. If changes in these running dynamics can be observed then on-board estimation of the adhesion level in real time is possible.

The method employed to estimate adhesion is a model-based approach using a linear suspension model as the basis of an estimator [3]. The use of this method on rail vehicles is not limited to only adhesion measurement. Model-based approaches have been suggested for suspension condition monitoring [6], suspension parameter modelling $[7,8]$, suspension fault detection $[9,10]$ and wheel/rail condition monitoring [5]. It has been shown in [11-13] that there are wider opportunities for model based methods for parameter and/or estimation for fault detection on board a rail vehicle.

This paper discusses the results of multibody simulation (MBS) testing where the wheel/rail contact algorithm is modified to incorporate the latest thinking of adhesion at micro-slip [14]. This simulation model is then used as a baseline to produce a suitable abstracted design model of a railway vehicle in order to create an effective model-based state estimator of creep forces for varying adhesion levels. Finally, the estimator is tested and assessed using the modified high fidelity multibody simulator VAMPIRE [15]. The use of a MBS is considered a key milestone in validating the suggested technique as the dynamic quantities generated are close to those experienced by a true rail vehicle. As further verification of the technique a series of tests were performed where only simulated inertial measurement data was initially provided in order to create a 'blind' approximation of adhesion.

\section{Low Adhesion Characteristics at Microslip and Incorporation in the 'Vampire' MBS package}

Previous studies of low adhesion at micro-slip using a tribometer train, [16], suggested that the initial creep slope decreases for lower adhesion levels, in addition to the expected reduction of the saturation level. This means that variations in the adhesion level manifest in different dynamics of a railway vehicle in normal or unsaturated creep force running. This is contrary to the established method of viewing creep forces, [17], which suggests dynamic differences due to adhesion variations only occur as the creep forces near saturation. It has since been established in laboratory conditions that this change in the initial creep slope is a physical phenomenon that is quantifiable for different friction modifying materials such as: water; oil; and simulated leaf contaminant [14]. Figure 1 shows a derived set of creepage/creep force curves for 4 generic adhesion conditions defined in Table 1 . These levels were chosen as they represent the transition between different levels of operational risk during normal running.

The MBS package VAMPIRE was modified to incorporate the latest thinking of creep force approximation at microslip conditions. The previous model based on the idea that creep force change due to changing adhesion is only evident at saturation is replaced by creep curve data presented in Figure 1, with appropriate 


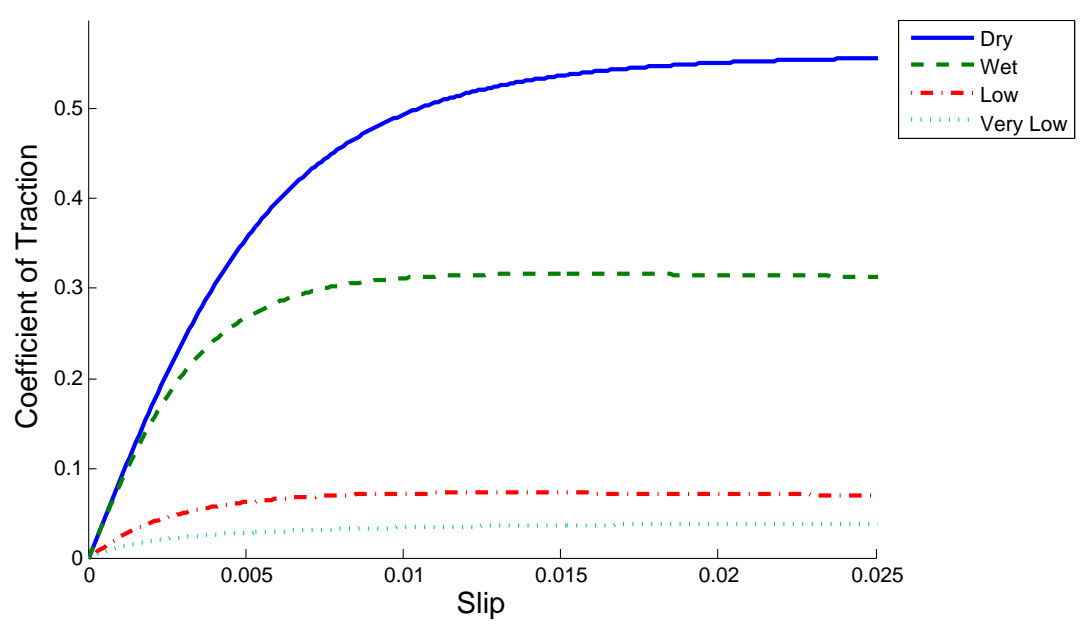

Figure 1. Figure showing changing initial creep slope with respect to reduction in adhesion levels

interpolation algorithms. This creates a new simulation model that will be used as a baseline for both the production of an abstracted design model of for use in a model-based estimator, and for the testing of this estimator by producing simulated inertial data.

\section{Model Abstraction for Creep Force Estimation}

The modification of the VAMPIRE MBS described in section 2 provides verification opportunities for creep force estimation, but challenges remain to produce a model-based estimator capable of operating at extreme levels of low adhesion. It has been shown $[18,19]$ that the dominant stability characteristics with regard to the hunting motion of the rail vehicle can be captured by modelling only the lateral and yaw component dynamics. The technique presented in [3] uses this so called linear plan-view representation as the basis of a model-based creep force estimation technique. Deriving a simplified plan-view model that correlates well with a MBS model (and by extension real rail vehicles) is a far from trivial process. The assumptions made for modelling a rail vehicle operating at normal operating conditions have to be challenged to confirm they remain true, especially at extremely low levels of wheel/rail adhesion.

\subsection{The Restorative Reaction Force in Conditions of Extreme Low Adhesion}

Studying the results obtained from VAMPIRE simulations, it was found that the restorative force due to lateral displacement as a result of the vehicle weight and the wheel/rail contact angle, often called gravitational stiffness, became an increasingly dominant term with respect to the creep force magnitudes as the adhesion level reduced. This is shown in Figure 2 where the gravitational stiffness is plotted alongside the total sum of the lateral contact forces acting on the wheelset. The total lateral force exerted on the wheelset $F_{w y}$ can be expressed as the sum of the lateral creep force $F_{c y}$ and the gravitational stiffness $F_{g}$.

$$
F_{w y}=F_{c y}+F_{g}
$$

This data is taken from two VAMPIRE simulations of a typical modern style 
passenger diesel multiple unit (DMU) at the dry and very low adhesion conditions. The vehicle chosen has a contemporary suspension design consistent with modern vehicles and lends itself well to a linear model. In these tests the vehicle is modelled as travelling at $50 \mathrm{~m} / \mathrm{s}$ along a track with irregularities representative of those found on high-speed lines within the UK. It can be seen that the gravitation stiffness term
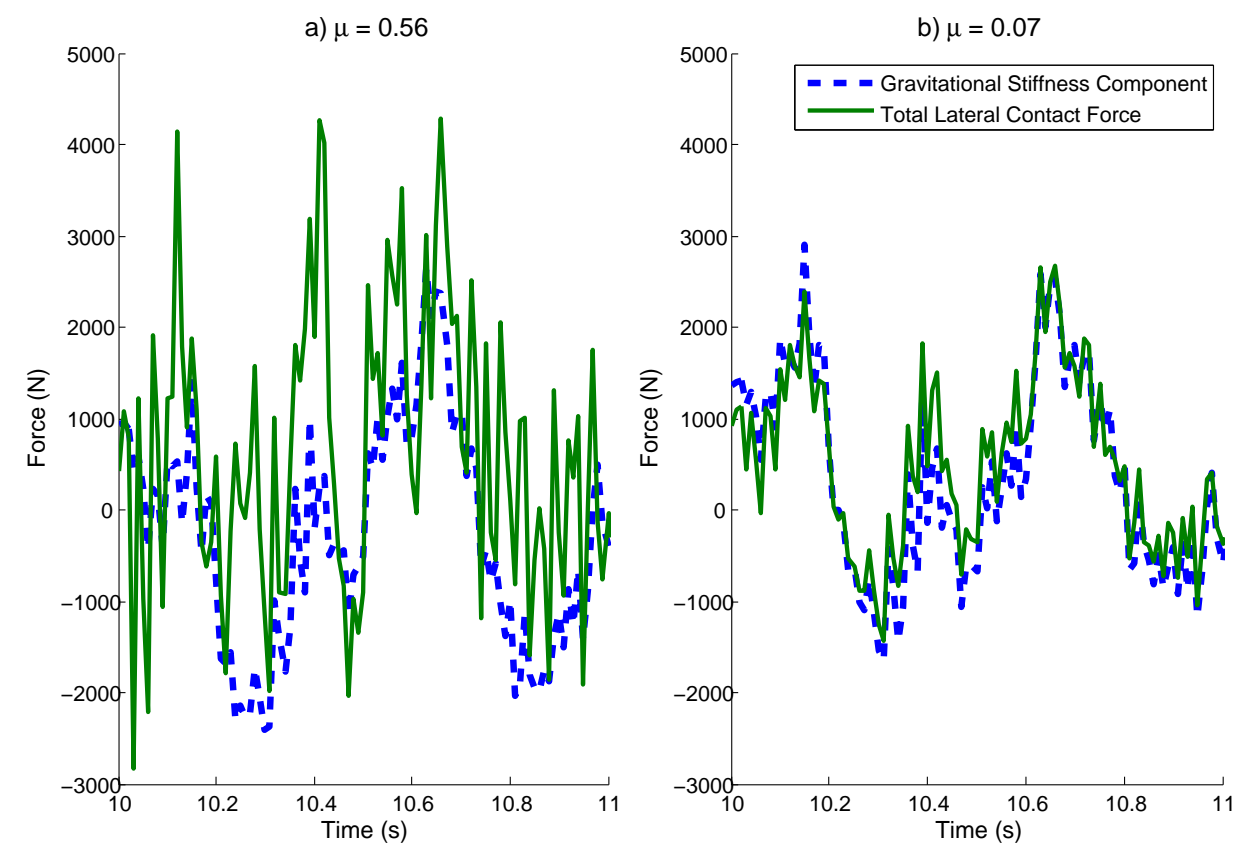

Figure 2. Comparison of Lateral Wheelset Forces at a) 'dry' and b) 'very low' adhesion conditions

becomes the dominant restorative force component at very low adhesion conditions. In dry conditions, the magnitude of the creep force is such that it masks any modelling errors that may be present with the gravitational stiffness term meaning a linear geometric description of the wheel/rail profile can be used in most higher level adhesion circumstances. However to facilitate very low adhesion conditions a linear approximation may no longer be satisfactory and greater care is required to include the non-linear geometries in the contact patch.

To form a mathematical description of the problem, consider the forces acting on a conical wheel in the vertical and lateral directions, as shown in Figure 3. In this Figure, the resultant reaction force due to the weight acting through the wheel set is shown as $R$ and has an angular offset to the vertical direction due to the contact angle $\delta$. The resolved gravitational stiffness in the ' $y$ ' plane is shown as $F_{G R}$. The creep force experienced by the wheelset due to the lateral displacement $y_{w}$ is shown as $F_{C R}^{\prime}$, and is resolved to the ' $y$ ' plane as $F_{C R}$. Because the conicity of the wheel normally represents a small angle, it can be said that $F_{C R}$ is equal to $F_{C R}^{\prime}$. Referring back to equation (1), the total gravitational stiffness $F_{g}$ acting on the wheelset in the lateral direction is the sum of the gravitational stiffness terms in the of the right and left wheel.

$$
F_{g}=F_{g L}+F_{g R}
$$

if the wheelset is subject to a load of $W \mathrm{~kg}$ and the contact angles for the left and right wheel $\delta_{L}$ and $\delta_{R}$ are small, equation (2) can be expressed as

$$
F_{g}=R_{R} \sin \left(\delta_{L}+\phi\right)-R_{L} \sin \left(\delta_{R}-\phi\right)
$$




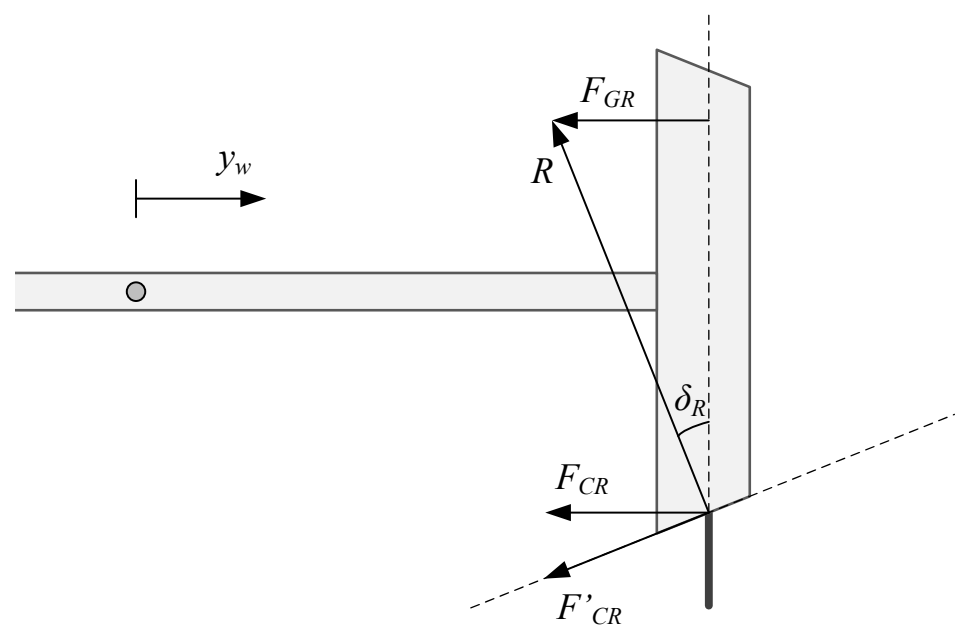

Figure 3. Contact forces experienced by a conical wheel on a simple rail. 'R' is introduced to the subindices to depict 'right'.

Where $\phi$ is the roll angle of the wheelset, caused by a difference in height between the right and left hand wheel. Equation (3) can be reduced further and expressed in terms of the weight acting through the wheelset.

$$
\begin{aligned}
& F_{g}=\frac{W}{2} \sin \left(\delta_{L}+\phi\right)-\frac{W}{2} \sin \left(\delta_{R}-\phi\right) \\
& F_{g}=W\left(\frac{\delta_{L}-\delta_{R}}{2}+\phi\right)
\end{aligned}
$$

It is commonplace to make initial modelling assumptions by defining the contact angles to be constant and dependant only on the conicity of the wheelset. For normal running in good adhesion conditions, this assumption will provide valid and usable approximations for estimating the motions of a wheelset [19]. When this assumption is expressed mathematically, the contact angles $\delta_{L}$ and $\delta_{R}$ are equal and opposite, cancelling each other out. This means when linear approximations are made, $F_{G}$ is solely dependant on the approximation of roll. If it is assumed the track is level (i.e. has a cant angle of zero) the roll angle is found from the difference in rolling radii of the wheels $r_{L}$ and $r_{R}$, and half the gauge width, $a$.

$$
\phi=\frac{\left(r_{L}-r_{R}\right)}{2 a}
$$

It can also be shown that equation (5) can be expressed in terms of the wheel conicity $\lambda[19]$.

$$
\phi=\frac{\lambda}{a} y_{w}
$$

To investigate the contact mechanics and assess the modelling assumptions, a simple rail vehicle model is introduced. In this case, a single wheelset is attached directly to a simple mass via springs and dampers in both the lateral and yaw directions with constants $k, k_{\psi}, b$ and $b_{p s i}$ respectively. The mass is considered fixed in yaw but free to move laterally. The wheel rail contact is based on linear Kalker creep laws and a linear approximation of gravitational stiffness. The model considered only the lateral and yaw dynamic responses of the components. The 
wheelset dynamic equations are given by:

$$
\begin{aligned}
m_{w} \ddot{y}_{w} & =F_{g}+F_{c y}+F_{\text {sus }} \\
& =W \frac{\lambda}{a}\left(y_{w}-y_{d}\right)-\frac{2 f_{22}}{V}\left(\dot{y}_{w}-V \psi_{w}\right)-\left(y_{w}-y_{m}\right) k-\left(\dot{y}_{w}-\dot{y}_{m}\right) b \\
I_{w x} \ddot{\psi} & =a F_{c R x}-a F_{c L x}+M_{\text {sus }} \\
& =-\frac{2 f_{11}}{V}\left(\frac{\lambda\left(y_{w}-y_{d}\right)}{a}\left(y_{w}-y_{d}\right)-a \dot{\psi}\right)-\psi_{w} k_{\psi}-\dot{\psi}_{w} b_{\psi}
\end{aligned}
$$

where minor terms have been removed. Here, $y_{m}$ and $\dot{y}_{m}$ are the position and velocity of the mass, $m_{w}$ is the weight of the wheelset with $I_{w x}$ the moment of inertia, $F_{\text {sus }}$ and $M_{\text {sus }}$ are the resultant suspension forces and moments. $V$ is the vehicle forward speed, $F_{c x r}$ and $F_{c x l}$ are the longitudinal creep forces of the right and left wheel, and $f_{11}, f_{22}$ are linear Kalker coefficients [19].

This vehicle was subject to a 5 second transit along a straight section of track that included a single $5 \mathrm{~mm}$ lateral step after 1 second of travel. This size step input was chosen as its magnitude falls within an expected size of irregularity on standard UK high speed lines. The simulations were repeated at dry and very low levels of adhesion.

Figure 4 shows the time history of $y_{w}$ and $\psi_{w}$ for this transit at both dry and very low adhesion conditions. These responses are compared to a VAMPIRE simulation of a similar model with the exceptions that this, considers the six degree-of-freedom motion of the wheelset; has a non-linear approximation of creep force; has a nonlinear approximation of gravitational stiffness.

It can be seen by visual inspection of the plots that at dry conditions the dynamic response of the models match well. It would be reasonable to assume that a linear approximation is representative of the more complex simulation model. In the very low adhesion conditions it is clear that the models do not match well. It was found that the contact forces in the linear model were considerably lower than in the VAMPIRE model, so the wheelset response in the linear model is considerably slower to follow the step change in the track. It is clear that a linear approximation in this case is not representative.

\subsection{Linear versus non-Linear Contact Approximations}

Figure 2 demonstrated that the primary restorative force to the wheelset alignment with the rail is provided by the gravitational stiffness in conditions of very low adhesion. The previous section highlighted the shortcomings of using a linear approximation to model the gravitational stiffness term. This section investigates the changes that have to be made to the linear assumptions in order to accurately model the contact forces at low adhesion levels in open loop models.

One of the main assumptions made is approximating a rail wheel as a conical shape in contact with a 'knife-edge' rail. As can be seen in Figure 2, this approximation is reasonable for good adhesion conditions under normal running. A true wheel is not conical and has a curved profile, as shown in Figure 5. In addition, the rail head itself is not a single point but also has a profile. The contact angles $\delta_{L}$ and $\delta_{R}$ are non-trivial as they depend on the profile of the wheel, the rail and the lateral displacement between the two.

The assumptions that were made to simplify the expression for gravitational stiffness in equation (4) are no longer applicable. Instead, gravitational stiffness can be shown to be: 

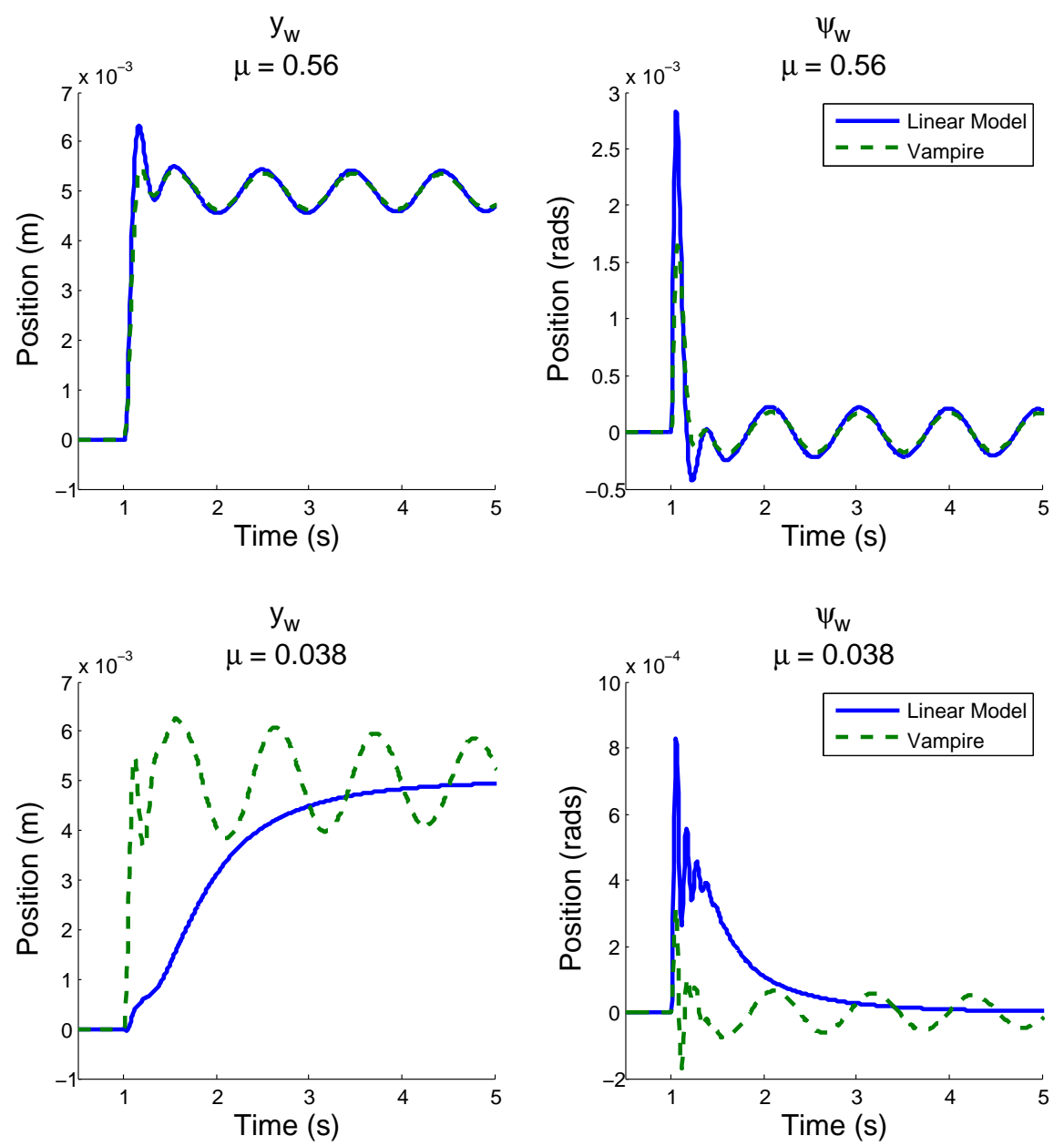

Figure 4. Comparison of Linear, plan-view model to a VAMPIRE simulation of a single wheelset

$$
\begin{aligned}
& F_{g}=W\left(\frac{\delta_{L}-\delta_{R}}{2}+\phi\right) \\
& F_{g}=W\left(\frac{\delta_{L}-\delta_{R}}{2}+\frac{\left(r_{L}-r_{R}\right)}{2 a}\right)
\end{aligned}
$$

In order to model the non-linear contact geometry, the contact angles $\delta_{L}$ and $\delta_{R}$ along with the rolling radii $r_{L}$ and $r_{R}$ are obtained using linear interpolation as a function $y_{w}-y_{d}$. The interpolation tables are populated using known profile results for a 113A railhead and a $\mathrm{P} 8$ as shown in Figure 5.

Using this non-linear description of the contact geometry in equation 7 , the single wheelset vehicle model used to generate Figure 4 can be repeated to assess the effects on gravitational stiffness. The results of this simulation are shown in Figure 6 and are plotted alongside the previous results assuming linear contact geometry.

Figure 6 shows that the response of the linear plan-view vehicle model matches well to the VAMPIRE simulation when a non-linear $F_{g}$ term is included, even in conditions of very low adhesion.

Figure 7 shows the contact forces experienced during the simulation using the non-linear contact description. Over-laid on this plot is the gravitational stiffness experienced to highlight the proportion of the overall contact force. Also shown is 


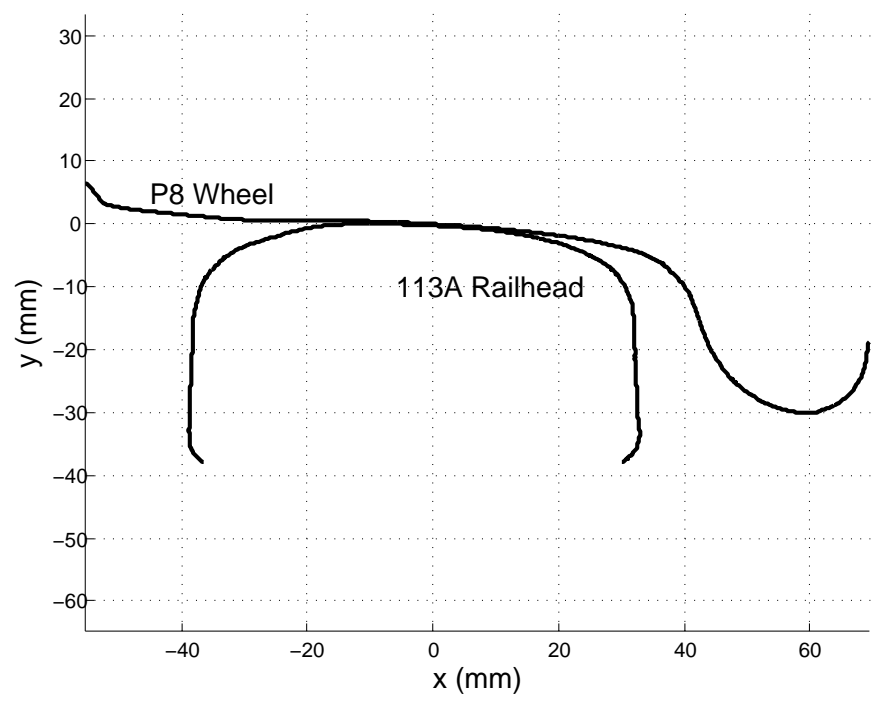

Figure 5. A profile of a P8 wheel and a 113A Railhead

the gravitational stiffness approximation should a linear approximation had been taken at that instance in time.

Figure 7 highlights that the linear approximation method always underestimates the value of gravitational stiffness, regardless of the level of adhesion. It also highlights that the gravitational stiffness term is the dominant stabilising force in the very low adhesion condition compared to being a very minor term with the dry condition.

The findings presented in this section highlight two main points:

- The linear approximation of creep force (using Kalker coefficients) aligns well with the non-linear description with VAMPIRE, even at conditions of very low adhesion

- $F_{g}$ is defined by the wheelset geometry and a generic, conical approximation is not valid in low adhesion conditions for open loop models.

A linear plan-view model defining motions in the lateral and yaw direction should be an appropriate method for modelling more complex suspension arrangements and capturing the vehicle stability motions. However, to validate this as a development model in open-loop tests in very low adhesion conditions, non-linear contact laws to simulate gravitational stiffness must be applied.

\subsection{Development of Model for Creep Force Estimation}

The technique of using a model-based approach to estimate creep forces and adhesion has been demonstrated as a viable technique in both linear suspension model scenarios [5, 20] and recently with more complex suspension models [21] in low level nonlinear simulation modelling. This approach requires a well validated linear plan-view vehicle suspension model to provide accurate results.

When modelling the dynamic response of the wheelset and bogie at reduced levels of adhesion, extra care is required for the mathematical description of the suspension components. It is often the case, that due to the reduced contact forces, only a small amount of displacement in the suspension components is experienced as a result of track irregularities. This results in suspension components operating outside of their expected behaviour profiles and previous linear assumptions no longer apply. This can prove troublesome when attempting to produce a simplified 

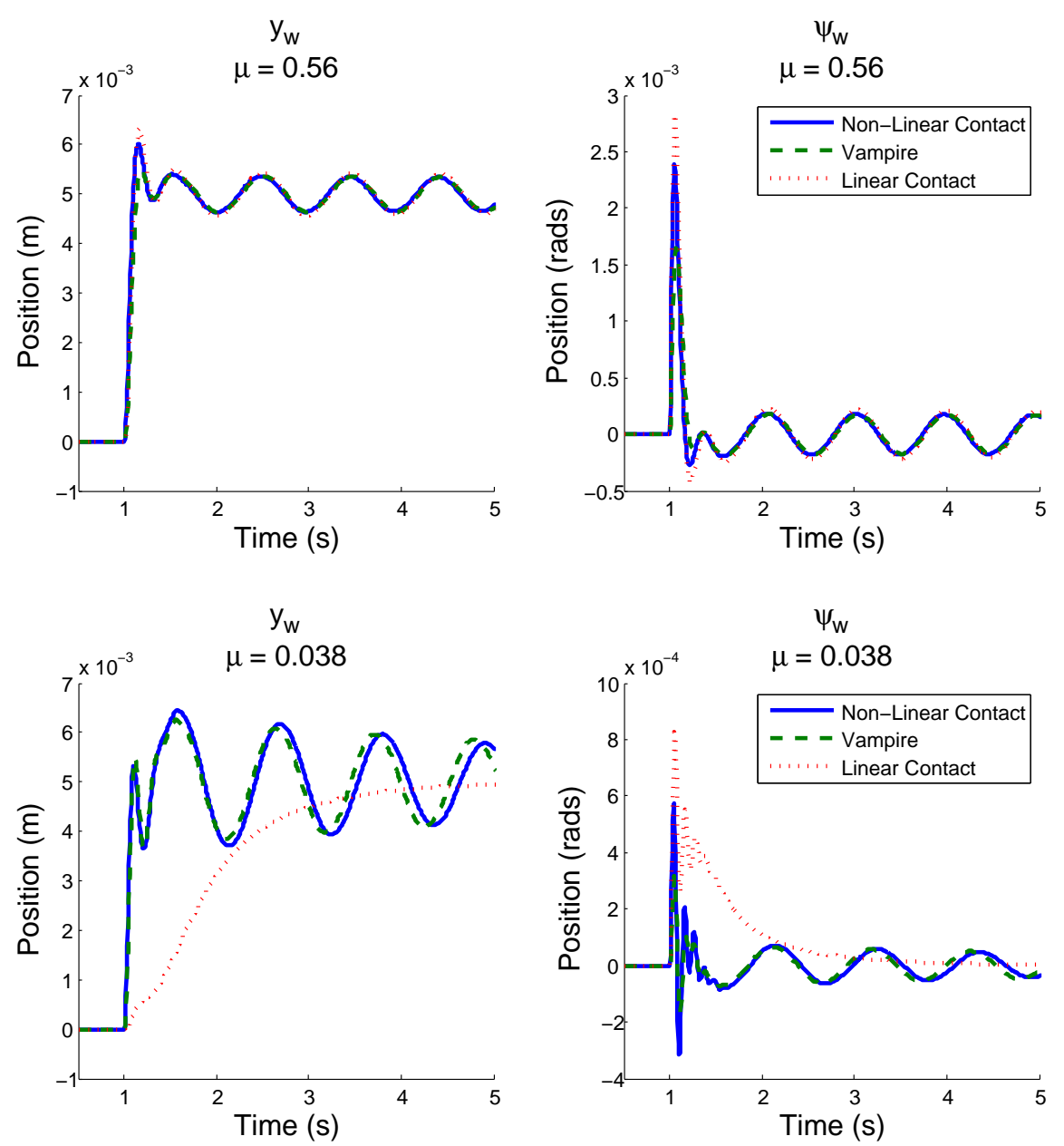

Figure 6. Comparison of VAMPIRE simulation to linear $F_{g}$ approximation with a linear plan-view model to non-linear $F_{g}$ approximation with a linear plan-view model.

linear model for estimation purposes.

As with the single wheelset model in section 3, A dynamic system model for this half vehicle development model is generated by developing a series of linear differential equations considering the Newtonian force/mass balance relationships of each of the wheelsets, bogies and vehicle body. For the purposes of designing a model-based estimator it is necessary to produce a half vehicle model that describes the lateral and yaw motions of the front wheelsets, bogie and vehicle body. Similar models have been produced in previous work [5, 22].

Figure 8 shows the layout of the suspension components considered for the half vehicle model. This system is chosen to represent a generic modern passenger DMU with a contemporary bogie design. The parameters used to represent this vehicle can be found in Appendix A.

This reduced model can be validated in open loop tests against a VAMPIRE simulation that considers non-linear components modelled in six degrees of freedom. In order to validate this model in low adhesion conditions, the non-linear contact description described in section 3 must be used.

Each model is subject to the same lateral step input of $5 \mathrm{~mm}$ and the time response of the wheelset position in both yaw and lateral displacement is shown in Figure 9. The simulation is performed at the most testing conditions of both low and very low adhesion levels.

It can be seen that the linear model provides a good representation of the VAMPIRE MBS at the low adhesion level as it accurately captures the significant modes 

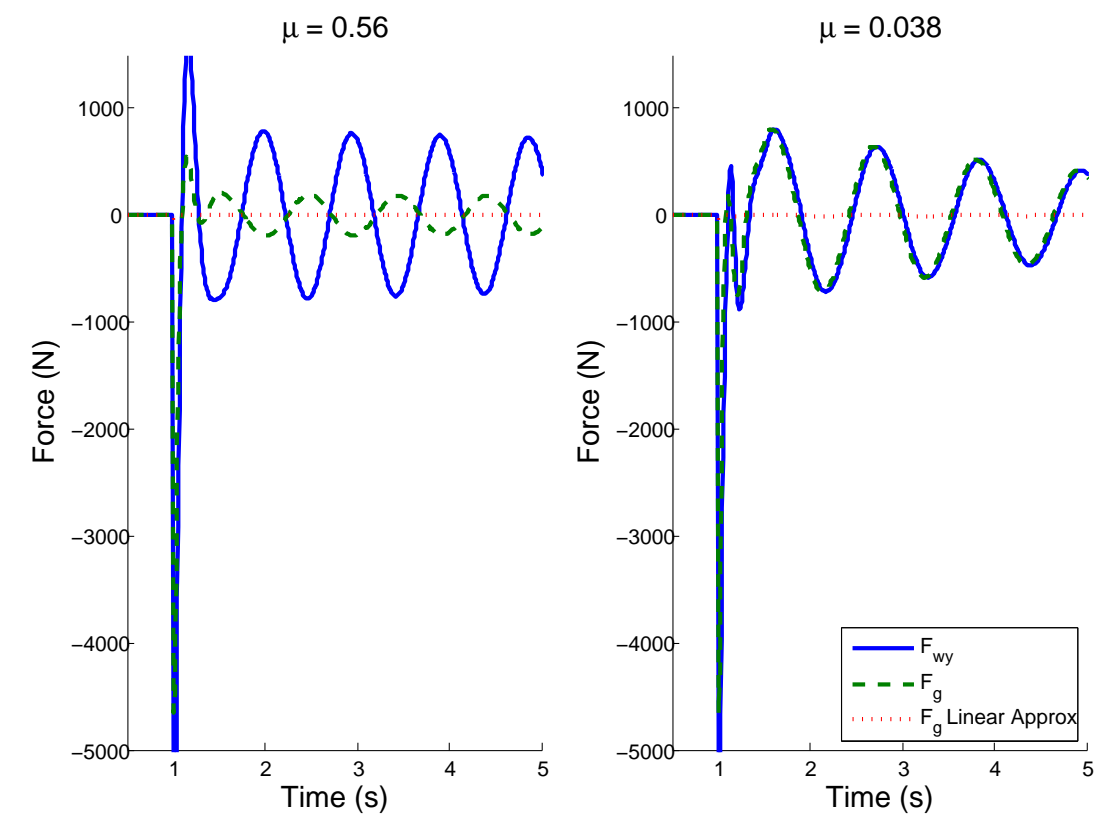

Figure 7. Comparison of Total lateral contact force $F_{w y}$ to gravitational stiffness $F_{g}$ and a linear approximation of $F_{g}$ at a) Dry conditions and b) Very low adhesion conditions.

a)

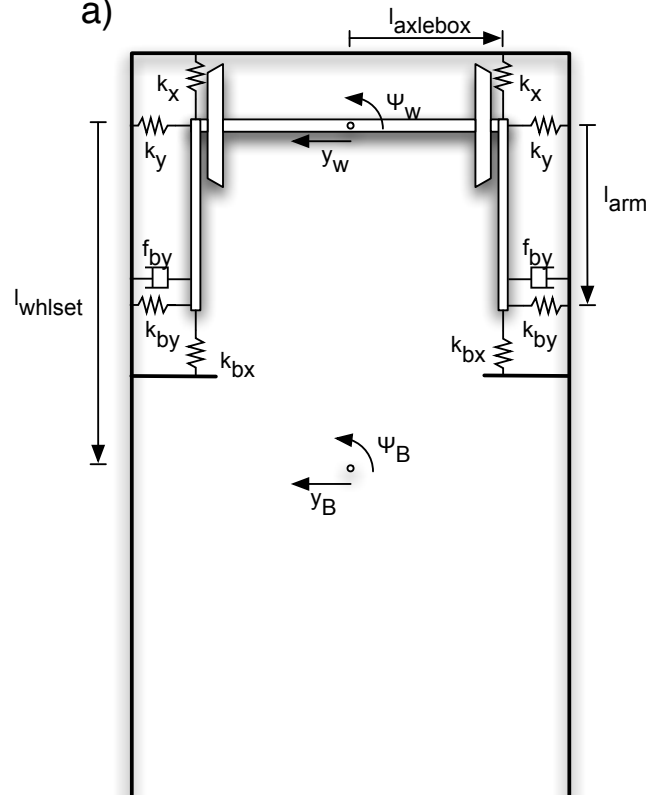

b)

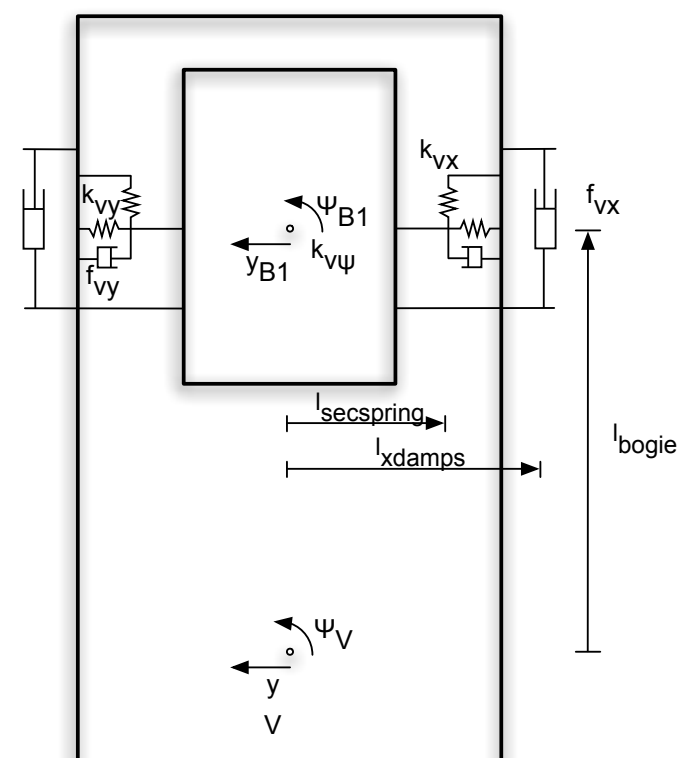

Figure 8. Schematic of a linear plan-view model of a half rail vehicle suspension when considered in lateral and yaw ranges of motion. a) shows the primary suspension, b) shows the secondary suspension

experienced by the system. As expected, the largest discrepancy observed is at the very low adhesion levels where the linear approximation cannot represent friction that has not been broken out or other non-linear elements of the suspension. It does however provide a good enough representation to verify the efficacy of the suspension model for use in a model-based estimator as in [3].

\section{Model-Based Creep Force and Adhesion Level Estimation}

This method uses a Kalman-Bucy filter based on the linear plan-view suspension model of the rail vehicle. By monitoring the associated vehicle dynamic quantities, 

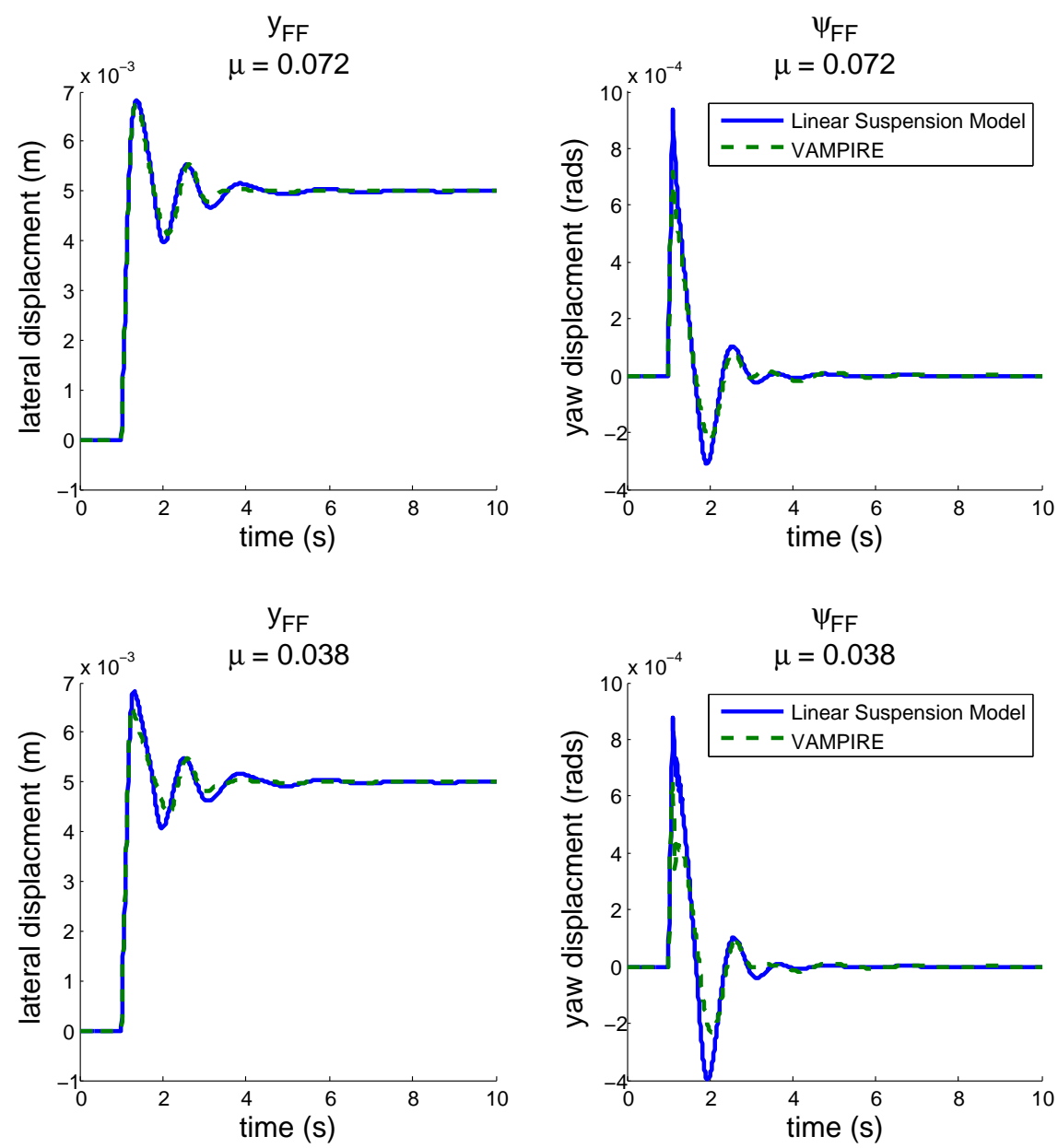

Figure 9. Comparison of the half vehicle linear suspension model simulation to VAMPIRE simulation at low and very low adhesion conditions

the contact forces can be estimated as augmented states.

$$
\begin{aligned}
& \dot{\mathbf{x}}=\mathbf{A}_{\mathbf{k}} \mathbf{x}+\mathbf{B}_{\mathbf{k}} \mathbf{u} \\
& \mathbf{y}=\mathbf{C}_{\mathbf{k}} \mathbf{x}+\mathbf{D}_{\mathbf{k}} \mathbf{u}
\end{aligned}
$$

In this application, the states chosen to populate the state vector $\mathbf{x}$ are the leading wheelset lateral and yaw position and velocity, along with the total lateral contact force and total contact moment in yaw experienced by the wheelset.

$$
\mathbf{x}=\left[\begin{array}{llllll}
y_{w} & \dot{y}_{w} & \psi_{w} & \dot{\psi}_{w} & F_{w y} & M_{w \psi}
\end{array}\right]^{T}
$$

The use of this method is restricted in that it is not possible to distinguish between creep force and gravitational stiffness [5] and as such they are grouped together as collective terms that represent the total contact force $F_{w y}$ and total contact moment $M_{w \psi}$ experienced by the wheelset and are given by:

$$
\begin{aligned}
F_{w y} & =F_{c y}+F_{g} \\
M_{w \psi} & =a F_{c R x}-a F_{c L x}
\end{aligned}
$$


Other terms do exist that can contribute to the total contact moment experienced by the wheelset, but these are found to be very minor in comparison to the longitudinal creep forces, even in conditions of very low adhesion.

To simplify the structure of the estimator, the secondary suspension is excluded from the model-based filter by defining the bogie positions and velocities as inputs to the system. The input vector is defined as

$$
\mathbf{u}=\left[\begin{array}{llll}
y_{B} & \dot{y}_{B} & \psi_{B} & \dot{\psi}_{B}
\end{array}\right]^{T}
$$

The state and input matrices $\mathbf{A}_{\mathbf{k}}$ and $\mathbf{B}_{\mathbf{k}}$ are populated using the linear description of the suspension model introduced in Section 3.3.

The Kalman-Bucy filter is able to provide an estimate of the system states using a mathematical approximation of the current state, plus an adjustment based on measurements within the system. This is expressed by:

$$
\begin{aligned}
\dot{\hat{\mathbf{x}}} & =\mathbf{A}_{\mathbf{k}} \hat{\mathbf{x}}+\mathbf{B}_{\mathbf{k}} \mathbf{u}+\mathbf{K}(\mathbf{y}-\hat{\mathbf{y}}) \\
\hat{\mathbf{y}} & =\mathbf{C}_{\mathbf{k}} \hat{\mathbf{x}}
\end{aligned}
$$

The output matrix $C_{k}$ is defined to make all of the states appear as outputs in the estimated output vector $\hat{\mathbf{y}}$. The adjustment made to the state estimation is performed by multiplying the difference between the estimated outputs in $\hat{\mathbf{y}}$ and the measure outputs $\mathbf{y}$ multiplied by the Kalman gain matrix $\mathbf{K} . \mathbf{K}$ is derived via an optimisation technique that relies on specifying a degree of certainty with each of the state models. In this implementation, the contact force and moment are defined as being fundamentally uncertain with respect to the positions and velocities. Therefore contact force is estimated based on the residual values of measured and estimated state outputs and the mathematical model of the suspension.

\subsection{Validation of Creep Force Estimation using VAMPIRE}

In order to prove the efficacy of the contact force estimation technique a series of 'blind' test data were created. In these tests, inertial data generated from a VAMPIRE simulation of the generic passenger DMU was provided in order to estimate the contact forces. These 60 second long simulations were performed at constant vehicle velocity and upon track containing irregularities typical to those found on UK high speed lines. The actual levels of adhesion used in the simulation, along with track measurement were withheld. The inertial data generated was input into the Kalman-Bucy filter designed in the previous section and the contact forces estimated were recorded.

A 2.5 second snapshot of the results are presented in Appendix B. It can be seen that the contact force estimation produces good agreement to the VAMPIRE simulation for creep moment at adhesion levels of $0.23,0.30$ and 0.07 (Runs 1, 3 and 4). Run 2, where the adhesion level is 0.04 , shows an overestimation of creep moment compared to the VAMPIRE simulation. This lack of resolution was expected for the reasons given in section 3.3. There is also some agreement in the lateral contact forces, but it is not as clear as the creep moment due to the higher frequency content.

These results are significant as they demonstrate the principle to provide realtime creep/contact force estimation of rail vehicles, based only on inertial sensor measurements. 


\section{Verification of Adhesion Estimation with 'Blind Data'}

A further challenge is to process the estimations of contact force into an adhesion estimate as it is this metric that describes the current running condition of the track. Large and small contact forces exist across all ranges of adhesion as a contributing factor is the amount of slip occurring between the wheel and rail, as well as the coefficient of friction that exists between the two. For example, a small amount of slip at dry levels of adhesion will validly induce a small creep force. However direct measurement of slip requires the differential position measurement of wheel to track position which is a costly and difficult item to observe. A previous study [21] has shown that over a 5 second moving window, an RMS value of estimated creep moment scaled by the wheelset yaw acceleration can be calibrated against known conditions to provide an indication of the level of adhesion.

Although the contact forces $F_{w y}$ and $M_{w \psi}$ could be predicted quickly and accurately, as shown in section 4.1, the conversion to adhesion is not straight forward. Figure 10 shows the estimation of adhesion level with time.

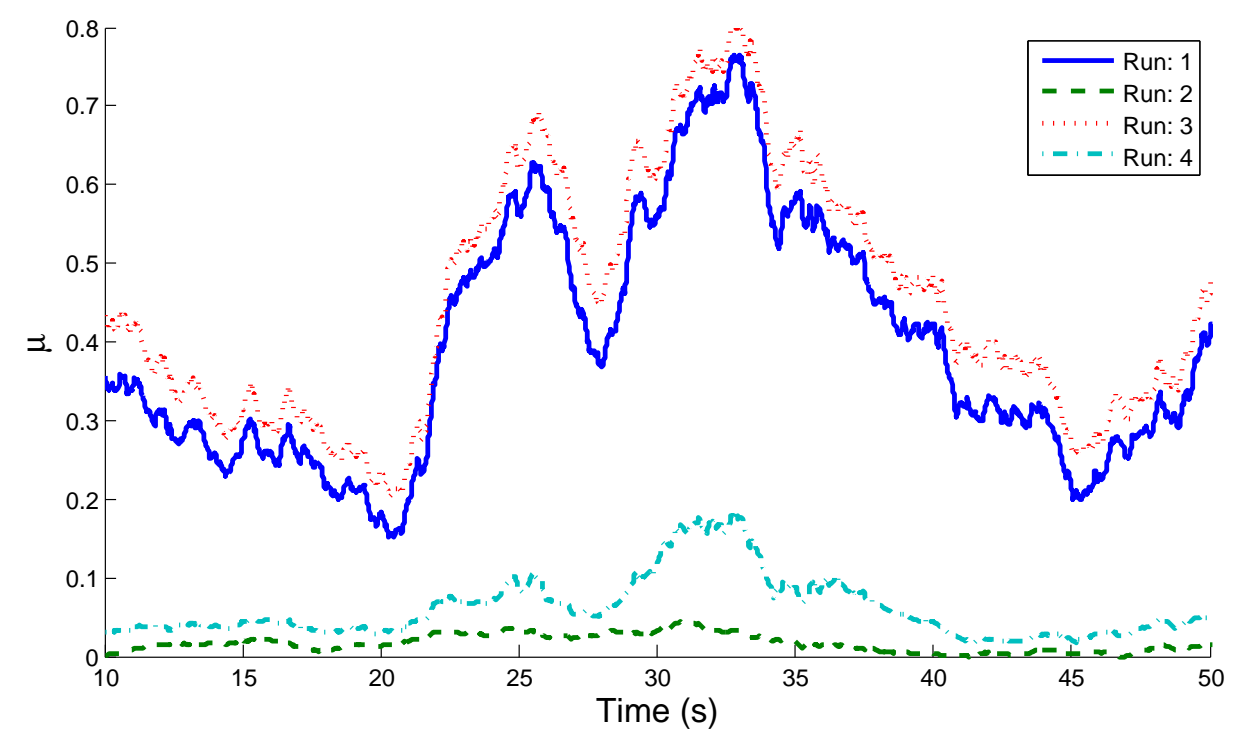

Figure 10. Estimation of adhesion levels experienced during four VAMPIRE simulations. The adhesion levels used are: Run $1-\mu=0.23$, Run $2-\mu=0.04$, Run $3-\mu=0.30$, Run $4-\mu=0.07$

The large variation in adhesion prediction in runs 1 and 2 is attributed to the variation in the level of track irregularities and the estimation of these using yaw acceleration. Furthermore, the calibration focusses on achieving accuracy at the lower levels of adhesion as this is the region where operational difficulties occur. This leads to an over-estimation of adhesion at higher levels of $\mu$.

The comparison of these estimated adhesion levels against the actual levels used in simulation is summarised in Figure 11. Here, the average estimated level of adhesion over the whole run is plotted against the level of adhesion used in the original MBS. The shaded areas represent different operational risk levels associated with normal running namely 'good', 'reduced' and 'poor'. The dashed line represents perfect estimation as points occurring here will represent cases where estimated adhesion equals actual adhesion.

Figure 11 shows that there is some discrepancy between the two values of adhesion in good operating conditions (where $\mu=0.23$ and $\mu=0.30$ ). In good adhesion conditions, the estimation technique tends to overestimate adhesion levels. At the lower levels of adhesion, it can be seen that the margin of error is smaller and the 


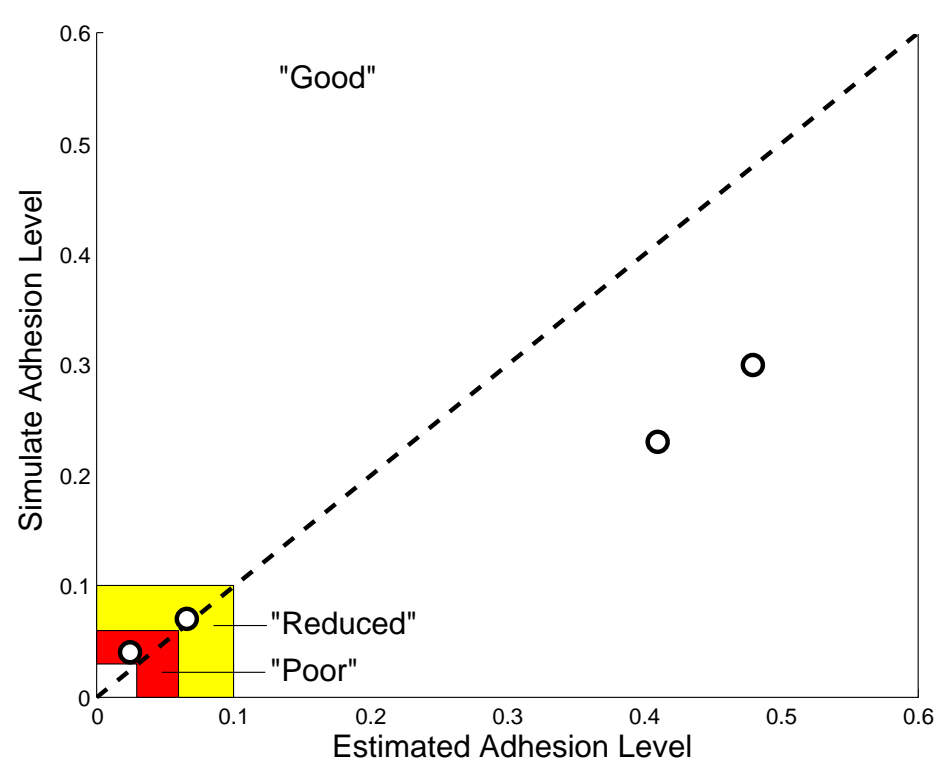

Figure 11. Figure showing estimated adhesion levels against adhesion levels used in simulation

estimator closely matches the value of $\mu$. Some of this is due to the calibration technique used in that the focus is placed on differentiating between different risk levels at lower adhesion. In all cases the estimator correctly predicts the overall operating conditions (i.e. 'good', 'reduced' or 'poor') for each test run.

Initial studies suggest that the estimation technique can be further refined by filtering the estimated output into the region of 1-10 Hz. This would focus on outputs due to the predominant vehicle lateral and yaw dynamics and remove some noise attributed to the filtering technique. In turn, this reduces some of the large variation seen in adhesion estimation due the varying size of irregularity in the track.

\section{Findings and Further Work}

This paper has shown the importance of the extra considerations required when generating models that incorporate low adhesion between the wheel and rail. At these conditions the reduced gradient experienced in the creep/creep force curves results in the gravitational stiffness becoming the dominant force that corrects lateral displacement of the wheelset. Modelling this force requires careful consideration of the contact geometry experienced between the wheel and rail meaning linear approximations may no longer be valid.

Understanding this issue assisted in verifying a linear plan-view suspension model against high fidelity MBS in conditions of low/extremely low adhesion. This abstract model forms the basis of a model-based estimator used to approximate creep force, and in turn adhesion, during normal running. The results presented in this article show that it is possible to perform real time estimation of creep force in the wheel rail contact using only inertial measurements taken from the wheelsets and bogies. The blind data tests prove that this can be done with no knowledge of the track alignment or operating adhesion condition.

The creep-force estimation technique relies on the ability to accurately model a complex suspension arrangement in a two-dimensional, linear, plan-view arrangement. At low levels of adhesion, the non-linearities in the suspension components are more exposed as breakout forces are less likely to be exceeded. Some suspension 
systems will not be sympathetic to a linear plan-view model that is verifiable in low adhesion conditions.

Further verification against blind data has shown that with simple postprocessing of creep force, it is possible to attain an indication of adhesion with a small processing delay of around five seconds.

The adhesion estimation technique has shown its limitations due to the difficulty in approximating the magnitude of track irregularity during normal running. A good statistical indication of track irregularity experienced would mean that the contact forces estimated can be effectively interpreted as adhesion levels. The current solution introduces a fives second delay into the estimation of adhesion which has an impact on potential operational implementations. For example, the adhesion estimation in its current form could not inform a braking system to optimise stopping distances but it could be used to form a network wide picture of the current track condition.

\section{Acknowledgements}

The authors would like to thank the RSSB and the TSLG for commissioning the project and providing industry led guidance throughout. The authors would also like to thank DeltaRail for supporting model development and providing simulated data from VAMPIRE models.

\section{References}

[1] B. Rowell and W. Richardson, An Assessment of the Available Adhesion and Slip Risk for ERTMS, (2003),

[2] I. Hussain and T.X. Mei, Multi-Kalman Filtering Approach for Estimation of Wheel-Rail Contact Conditions, in UKACC International Conference on Control IET, Coventry, UK, 2010, pp. $459-464$.

[3] C.P. Ward, R.M. Goodall, R. Dixon, and G.A. Charles, Adhesion estimation at the wheelrail interface using advanced model-based filtering, Vehicle System Dynamics 50 (2012), pp. 1797-1816.

[4] G. Charles, R. Dixon, and R. Goodall, Condition Monitoring Approaches To Estimating Wheel-Rail Profile, in Proceedings of the UKACC International Conference on Control, Manchester, UK, 2006.

[5] G. Charles, R. Goodall, and R. Dixon, Model-based condition monitoring at the wheel-rail interface, Vehicle System Dynamics 46 (2008), pp. 415-430.

[6] H. Tsunashima and H. Mori, Condition monitoring of railway vehicle suspension using adaptive multiple model approach; .

[7] P. Li, R. Goodall, and V. Kadirkamanathan, Parameter estimation of railway vehicle dynamic model using Rao-Blackwellised particle filter, in Proceedings of the European Control Conference, 2003, pp. $1-4$.

[8] P. Li, R. Goodall, P. Weston, C. Seng Ling, C. Goodman, and C. Roberts, Estimation of railway vehicle suspension parameters for condition monitoring, Control Engineering Practice 15 (2007), pp. $43-55$.

[9] H. Fan, X. Wei, L. Jia, and Y. Qin, Fault detection of railway vehicle suspension systems, in Computer Science and Education (ICCSE), 2010 5th International Conference on, 2010, pp. 1264-1269.

[10] Y. Hayashi, H. Tsunashima, and Y. Marumo, Fault Detection of Railway Vehicle Suspension Systems Using Multiple-Model Approach, Journal of Mechanical Systems for Transportation and Logistics 1 (2008), pp. 88-99.

[11] S. Bruni, R. Goodall, T.X. Mei, and H. Tsunashima, Control and monitoring for railway vehicle dynamics, Vehicle System Dynamics 45 (2007), pp. 743-779.

[12] C.P. Ward, P.F. Weston, E.J.C. Stewart, H. Li, R.M. Goodall, C. Roberts, T.X. Mei, G. Charles, and R. Dixon, Condition monitoring opportunities using vehicle-based sensors, Proceedings of the Institution of Mechanical Engineers, Part F: Journal of Rail and Rapid Transit 225 (2011), pp. 202218.

[13] R.W.N. Gu, C. Pislaru, A. Ball, and F, Modern techniques for condition monitoring of railway vehicle dynamics, Journal of Physics: Conference Series 364 (2012), p. 12016.

[14] D.I. Fletcher, A new two-dimensional model of rolling-sliding contact creep curves for a range of lubrication types, Proceedings of the Institution of Mechanical Engineers, Part J: Journal of Engineering Tribology (2012).

[15] DeltaRail, VAMPIRE;

[16] T.G. Pearce and K.A. Rose, Measured Force-Creepage Relationships and their use in Vehicle Response Calculations, Vehicle System Dynamics 15 (1986), pp. 427-440.

[17] J.J. Kalker, On the rolling contact of two elastic bodies in the presence of dry friction, Dissertation, Delft University of Technology, 1967. 
[18] A. Wickens Fundamentals of Rail Vehicle Dynamics, Taylor \& Francis, 2003.

[19] V.K. Garg and R.V. Dukkipati Dynamics of Railway Vehicle Systems, Academic Press, INC. (London) LTD., 1984.

20] C. Ward, R. Goodall, and R. Dixon, Creep Force Estimation at the Wheel-Rail interface, in Proceedings of the 22nd International Symposium on Dynamics of Vehichles on Roads and Tracks Manchester Metropolitan University, Machester, 2011.

[21] P. Hubbard, C. Ward, R. Goodall, and R. Dixon, Real Time Detection of Low Adhesion in the Wheel/Rail Contact, Proceedings of the Institution of Mechanical Engineers, Part F: Journal of Rail and Rapid Transit (2013).

[22] C.P. Ward, R.M. Goodall, and R. Dixon, Contact Force Estimation in the Railway Vehicle Wheel-Rail interface, in Proceedings of the 18th IFAC World Congress International Federation of Automatic Control, Milan, Italy, 2011.

\section{Appendix A. Parameters Used in the Half Vehicle Model}

Table A1. Suspension Model Parameters

\begin{tabular}{ccc}
\hline Parameter/Variable & Definition & Value \\
\hline$k_{x, y}$ & Primary spring stiffness & $0.494 \times 10^{6} \mathrm{~N} / \mathrm{m}$ \\
$k_{b x}$ & Primary bush stiffness & $6.915 \times 10^{6} \mathrm{~N} / \mathrm{m}$ \\
$k_{b y}$ & Bush damping coefficient & $1.729 \times 10^{6} \mathrm{~N} / \mathrm{m}$ \\
$f_{b y}$ & Secondary lateral stiffness & $1.73 \times 10^{3} \mathrm{Ns} / \mathrm{m}$ \\
$k_{v y}$ & Secondary longitudinal stiffness & $0.118 \times 10^{6} \mathrm{~N} / \mathrm{m}$ \\
$k_{v x}$ & Secondary yaw stiffness & $0.118 \times 10^{6} \mathrm{~N} / \mathrm{m}$ \\
$k_{v \psi}$ & Secondary lateral dampers & $49.4 \times 10^{3} \mathrm{Ns} / \mathrm{rad}$ \\
$f_{v y}$ & Secondary longitudinal dampers & $29.6 \times 10^{3} \mathrm{Ns} / \mathrm{m}$ \\
$f_{v x}$ & Radial arm Length & $0.247 \times 10^{6} \mathrm{Ns} / \mathrm{m}$ \\
$l_{\text {axlebox }}$ & Centre of primary spring from wheelset centre & $1.0 \mathrm{~m}$ \\
$l_{\text {arm }}$ & Moment arm of wheelset centre from bogie centre & $0.5 \mathrm{~m}$ \\
$l_{\text {whlset }}$ & Centre of secondary spring from vehicle Centre & $1.3 \mathrm{~m}$ \\
$l_{\text {secspring }}$ & Centre of secondary longitudinal dampers from vehicle centre & $0.925 \mathrm{~m}$ \\
$l_{x d a m p s}$ & Bogie semi-span & $1.25 \mathrm{~m}$ \\
$l_{\text {bogie }}$ & Weight acting through the wheelset & $8.0 \mathrm{~m}$ \\
$W$ & Lateral position of the wheelset & $95.63 \times 10^{6} \mathrm{~N}$ \\
$y_{F F}$ & Angular Position of the wheelset in yaw & $\mathrm{m}$ \\
$\psi_{F F}$ & Lateral Position of the bogie & $\mathrm{rads}$ \\
$y_{B}$ & Angular Position of the bogie in yaw & $\mathrm{m}$ \\
$\psi_{B}$ & Lateral Position of the vehicle body & $\mathrm{rads}$ \\
$y_{V}$ & Angular Position of the vehicle body in yaw & $\mathrm{m}$ \\
$\psi_{V}$ & & $\mathrm{rads}$ \\
\hline
\end{tabular}


Appendix B. Creep Force Estimation
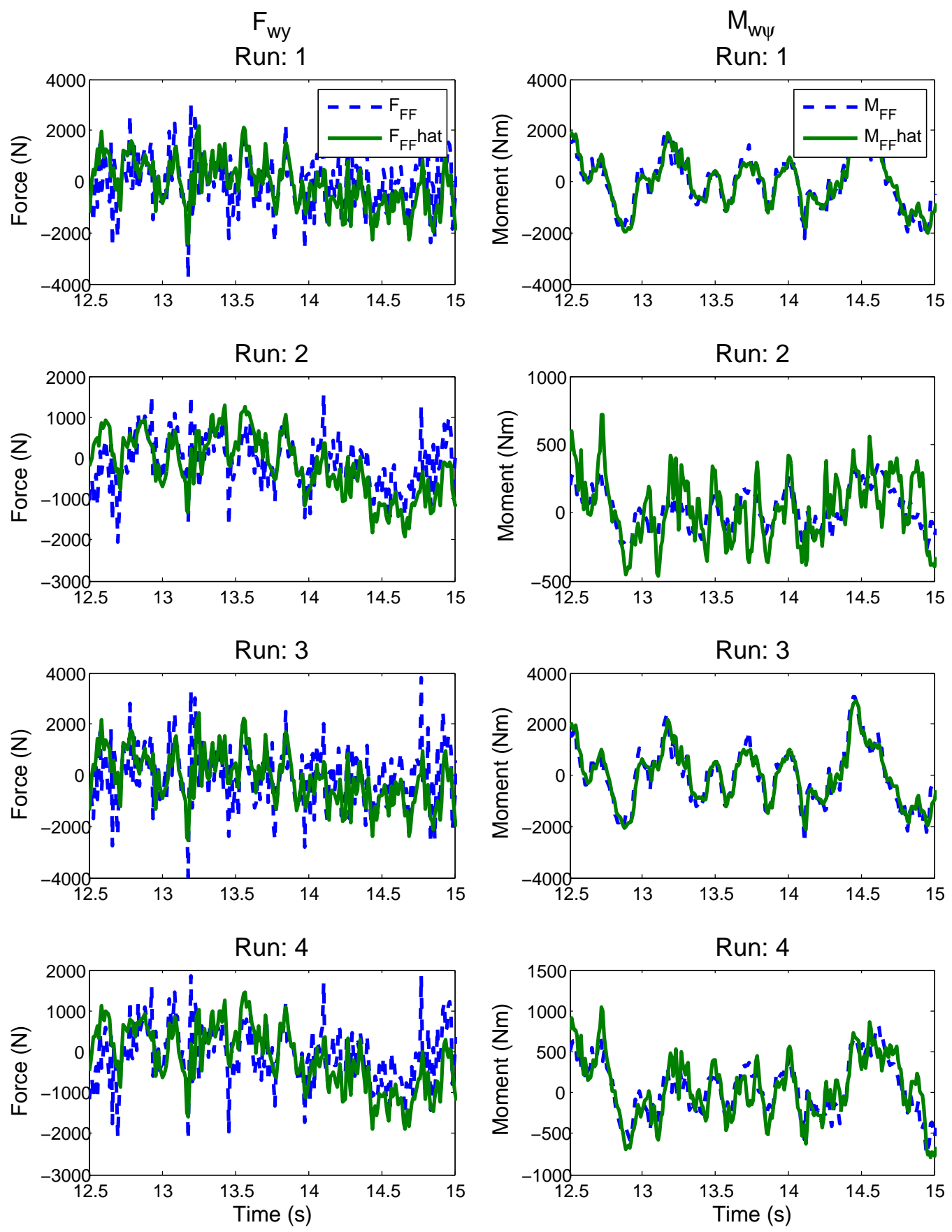

Figure B1. Time history plots showing simulated contact forces $F_{w y}$ and $M_{w \psi}$ against estimated contact forces. The values of adhesion for each simulation are: Run $1-\mu=0.23$, Run $2-\mu=0.04$, Run 3 $\mu=0.30$, Run $4-\mu=0.07$ 